\title{
I. Eröffnungsansprache
}

des Ersten Vorsitzenden, Geheimen Justizrats Prof. Dr. Heinrich Triepol.

Hoohgeehrte Herren! Liebe Kollegen!

Im Namen des Vorstandes der Vereinignng deutscher Stautsrechtolehrer habe ich die Ehre, Sie za Beginn anserer dritten Tagang berzlich willkomnen zu heiben. Wir freucu ans, daß Sie nuserm Rafe in stattlicher Zahl gefolgt sind. Zwar vermissen wir zu unserm Bedanern manchen aus der Reibe unserer Freunde, der anserer letzten Zasammenkunft in Jena beigewobnt hat. Besonders betrublich ist es mir, dab Altmeister $\mathrm{Ph}$ ilipp $\mathrm{Z}$ orn, der uns im vorigen Jahre durch seine Frische in Bewanderang versetzte, diesmal darch die Last des Alters verhindert ist, mit ans zusammenzotreffen. Einer unserer andern Senioreu, der von ans allen so huchrerebrte Wilbelm Kabl, hatte es nar gestern Abend moglich machen kounen, fur einige Standen unter ons zu sein. Er ist hente dorch eine wichtige Sitzung des Keichstags verhindert, in unserer Mitte zu weilen, und er hat mich ausdrlucklich beanftragt, Ihuen sein aufrichtiges Bedauern auszusprechen, dab er unserer Beratung fernbleiben maß. Dafur baben wir die Frende, eine Reihe von Mitgliedern, anch nen eingetretene, bei uns zu sehen, die zam ersten Male einer Tagang unserer Vereinigung beiwobnen. Ich rufe ihnen ein warmes Willkommen zu. Vor allem drängt es mich aber heute, verehrte Gäste za begrußen.

In erster Linie Seine Spektabilität den Herro Dekan und andere Mitglieder der Leipziger Jaristenfakultät. Wir danken ihnen ans vollem Herzen, da $B$ sie ans nach Leipzig eingeladen, daß sie die Muben der Vorbereitungen fur ansere Versammlaug ubernommen, and daß sie uns in gutiger Gastfreundschaft diesen schonen Saal ${ }^{1}$ ) als Stätte uuserer Arbeit zur Verfugang gestellt haben. Wollen Sie es, meine rerebrten Herren Kollegen, nicht als eine Abschweifung in das Gebiet des Allzapersönlichen betrachten, wenn ich als Kind der stadt Leipzig und als Sohn ibrel Juristischen Fukaltät dem Gefuble freudigen Stolzes daruber Ausdrack gebe, daB es mir vergount

1) Die Verhandlungen fanden im Collegium Juridicum statt. 
ist, hier in meiner Vaterstadt and in diesem Raume Ihre Versammlung zn leiten. Um dieses Haus weben sich fur mich zu viele Erinnerangen an bedentungsvolle Standen meines Lebens, als daß ich es mir versagen könnte, die Geister der eigenen Virgangenheit za beschwören. An diesem Tische habe ich meine erste Staatsprufang, dort druben im Sitzungszimmer der Fakultiit das Rigorosam bestanden - es sind beute wobl auf den Tag flinfunddreiBig Jahre her - dort babe ich den Doktoreid in die Hand Adolf Wachs abgelegt, and hier anf diesem Katheder habe ich meine erste akademische Vorlesung gehalti' $\mathrm{n}$. In Dankbarkeit und Webmut gedenke ich in dieser Stunde uer Miinner, die damals meine - und wie ich annehme, anch manches ron Ihnen - Lebrer gewesen sind, und die mich dann der Ehre gewurdigt haben, als Mitarbeiter in ihre Fakaltiit einzutreten, der Winds che id and $\mathrm{Sch}$ midt, der Binding und B 11 low, der Friedberg und Sohm. Ubi sunt qui ante nos in hac exedra foere? Nor einer ans dem Kreise der Mänuer, die damals die Zierde der I eipziger Fukaltät geweselı, wandelt noch im Lichte, aufrecht und geistesstark wie einst, frisch an Körper an!: an Seele. Wir haben' die große Freuile, ibn heute unter uns za sehen. Ich weib, meine Herren, dab ich Ihren lauten Beifall finden werde, wenn ich in Ihrem Namen Exzellenz Adolf W a c b, dem stolzen Zeugen einer der stolzesten Perioden der Leipziger Fakultiit, dem großen Prozessualisten, uns Staatsréchtslebrern besonders tener wegen der epochemachenden öffentlich-rechtlichen Grandlage, die er fur die Lehre des Zivilprozesses geschaffen hat, unsere verebrungsvolle Huldigang darbringe. (Lebhaftester Beifall.)

Meine Herreu! Die Leipriger Juristenfakultät, die uns heute so gastlich aufgenommen, bat nnserer $W$ i s 8 en s c h a f t nicht zu allen Zeiten eive wohleingerichtete Heimstätte ge:boten. Sie hat nicht die publizistische Tradition wie etwa Halle oder Göttingen oder Heidelberg. Noch in der Zeit, da ich bier stadierte, las der scharfsinnige $\mathrm{Karl} \mathrm{Vikt} \mathrm{or} \mathrm{Fricker}$ das Verwaltungsrecht, das Völkerrecht and selbst das Staatsrecht des Königreichs Sachsen als Professor der Philosophischen Fakultät. 'Erst mit 0 t t o I a y e r s Fintritt hat die Juristenfakultait einen Pablizisten im Hauptamte, freilich mit ihm sofort einen Mann ersten Ranges, gewonnen. Das Staatsrecht warde hier fruber immer nur von Gelebrten doziert, deren Hauptarbeit anderen Gebieten galt, von Germanisten, wie Wilbelm Edaard Albrecht and Karl Friedrich rou Gerber, ron Kanonisten wie Emil Friedberg, ron Kriminalisten wie $\mathrm{Karl} \mathrm{Bi} u \mathrm{ding}$. Und dennoch - wenn ich die Namen Albrecht and Gerber and Binding nenne, wie lebhaft tritt ans sofort vor die Seele, welche Fulle frachtbarer 
Anregang in Forschang and in Lehre fur das Staatsrecht schon in frtheren Zeiten von Leiprig ansgegangen ist! Das ist doch wobl anch ein Zeichen dafur, da $B$ ansere Wissenschaft inmer gat gediehen ist, wenn sie in lebendigem Zusammenbang gebalten worden ist mit der Geschichte des Rechts auf der einen Seite, anf der andern Seite aber mit ibren Schwesterdisziplinen, dem Strafrechte und dem ProzeB. Es mag trivial klingen, aber es verlobnt sich doch, hente vielleicht mebr als sonst, dab wir es sagen: die Rechtswissenschaft bleibt trotz aller Sonderung in einzelne Zweige immer eine einheitliche Wissenschaft, und so wertvoll es ist, wenn das Staatsrecht die Brucken zu Nachbargebieten, etwa der Gesellschaftswissenschaft, hinuberschlägt, seiner innersten Natur wird es untreu, sobald es sich eigenwillig ron dem Boden lost, aul dem es gewachsen ist. Der Kubm der Leipziger Jaristenfakultät aber ist es immer gewesen, daß sie dic Einheit des Rechto in allem Mannigfaltigen gewabrt bat. Wir danken es $\mathrm{ihr}$, daB sie auch in der Gegenwart mit sorglichen Händen das heilige Feuer butet, das uns alle mit der gleicben Glat erwärmt.

Ich habe ferner die Ehre, als unsere Gäste den Herrn P'räsidenten und eine Reibe ron Mitgliederu des Keichsgerichts za begrußen. Wir freuen uns, daß wir gerade beate einen Gegenstand bebandeln, von den wir hoffen können, er werde bei ibnen Interesse erwecken. Ich werde unsern Bericbterstattern vicht vorgreifen, wenn ich schon jetzt sage, $\mathrm{da} B$ das Reicbsgericht und seine Rechtsprechung in unserer Beratung eine grobe Rolle spielen wird. Wie es deno uberhaupt in seiver Judikatur fur uns Publizisten vou Jahr zu Jahr bedeatangsroller geworden ist! In ansern Tagen haben sich nicht nur Zivil- und Strafrechtslebrer, sondern auch die Männer des Staats- and Verwaltungsrechts dauernd in enger Fuhlang mit der Rechtsprechung des Reicbsgerichts za balten, and ich darf wobl behaupten, daß auch von uns jeder neue Band seincer Entscheidnngen mit Spaunung in die Hand genommen wird. Anch wir werden aufs stïrkste befruchtet ron den Anregungen, die von dort ansgeben, and selbst wenn sich gelegentlich einmal der Widersprnch des Theoretikers gegen eine Entscheidung des Praktikers regt, so sind doch auch wir Bewanderer der Sachlichkeit aller reicbsgerichtlichen Urteile and der Schärfe ihrer Begrundung. Mogen Sie, meine verehrten Herren rom Reichsgerichte, ans unserer Verbandlang die Gewißheit entnehmen, daB wir Lehrer des offentlichen Rechts in demselben Grade wie unsere Kollegen ans den anderen Kechtszweigen von der Ueberzeagang durchdrungen sind, es seien in Deatschland Praxis and Theorie des Rechts in einer anloslichen $\mathrm{E}$ b e verbunden. Und lassen Sie sich ferner sagen, was Ihnen 
in letzter Zeit schon oft gesagt worden ist: wie viel auch uns Dentschen die Jetzten Jahre genommen baben, eines ist uns nicht geraubt worden and kann uns nicht geranbt werden: die Znversicht, daß hier in Leipzig eine Stätte $h \delta \mathrm{chster}$ Gerechtigkeit erriohtet ist, bei einem Gerichtshofe, anf den Deutschland stolz ist, weil bei ihm, was Recht ist, anch immer Recht bleibt.

Hüchste Gerechtigkeit! Das ist ja auch das Ziel, dem wir alle, so versohieden unsere Wege sonst auch sein mogen, in gleicher Weise zustreben. Es ist anch das Ziel nnserer Beratungen an dieser Stelle. GewiB wird sich unsere Arbeit hente and morgen vielfach in der Kichtung anf eine $z$ w eck$m \ddot{a} B$ i g e Gestaltung rechtstechuischer Einrichtungen bewegen. Aber das bedentet ja doch keinen Gegensatz. Anf dem Gehiete des Rechts kann höchste Zweckmäßigkeit nichts anderes sein als höchste Gerechtigkeit. Denn die Gerechtigkeit ist und bleibt der oberste Zweck aller rechtlichen Ordnang. Die Gerechtigkeit ist daher anch die Herrscherin, der zn dienen und deren Reich za mehren, unser einziges Aint ist, und auser Bernf empfängt eber: tiierdurch seinen Adel. Möge der Throv uuserer Königin anch in diesem Saale aufgerichtet sein!

Mit diesem Wansche erkläre ich die diesjährige Tagung noserer Vereinigung fur eroffnet.

\section{Begrüissungsworte}

des Dekans der juristischen Fakultät der Universităt Leipzig, Geheinınn Justizrats Professor Dr. Alfred Schnltze.

Hochgeehrte Herren!

Fur die gutige Einladung zu Ibrer Tagung und die ebeu gehörten liebenswurdigen Worte lassen Sie mich den verbindlichsten Dank im Namen meiner Auftraggeberin aussprechen. Diese alte Dame ans gatem Hause, deren lhr Herr Vorsitzender. aus eigensten Erfahrangen schopfend, so herzlich gedacht bat, freat sich, ibre Räume den Herren fur ihre bedentangavolle Tagung offnen zo durfen. Gewiß ist sie stolz auf diesen Saal, der ibr altes Eigentum, und ibre Abnenbilder, die hente auf Sie herabschanen. Doch macht sie sich einige Sorge, ob er lhuen anch genugen wird. Sie befinden sich im lnnern eines Leipriger Geschäftshauses, in das zumeist selbst das liebe Hinmelslicht trub durch (nicht einmal gemalte) Scheibeu bricht. Ein Gluck wenigstens, daß die Sonne es in Angenblick be- 
sonders gut mit Ibnen meint nnd sich die grobte Muhe gibt, hinein zu strablen. Aber es durfte Sie auch anderes merkwirdig anmuten, wie etwa die alten Tische, deren Bloben and Schäden der Ortsansschu $\beta$ durch ein Gewand zu bedecken beschlob, das vielleicht gar za sehr noch an Papierserviette, Papierbindfaden, Pupiermark und an alle die anderen papiernen Errungenschaften der letztrergangenen Zeit erinnert. Dies alles soll nan durch die Wärme ausgeglichen werdev, mit der die alte Dame, die Leipriger Juristenfakaltiit, Ihoen durch mich ihren Willkommgrub entbietet.

Auf Ihre Vereinigung wird der Jurist, weB Zeichens er ist, nit Freude und Hotfnung blicken. Widmet sie sich doch den Grundlagen fur die Arbeit aller juristischen Fiicher. Icb will uar eins beransheben: die Art der $\mathrm{Kech}$ tse $\mathrm{t} z \mathrm{u} \mathrm{ng}$. An dieser sind wir alle interessiert. Wir wissen und fublen, dab hier gegenwïrtig vieles im Argen liegt. Es ist die bðchste Zeit, daB gegenuber der eingerissenen Praxis anf diesem Gebiete, man krunte auch ron "Praktik“ sprechen, das Statsrecht und die Stantsrechtstheorie zum Worte kommt und sich die dringend nötige Geltung verschafft. Ihre vorige Tugang in Jena hat sich bereits dieser Fragen angenommen, und wir Juristen alle sind lbren damaligen Beratungen mit großem Interesse gefolgt. Es wird noch weiterer Arbeit bedurfen Aber auch die Fragen, die auf Ihrer diesmaligen Tagesordnong stehen, beansprachen ein gleiches allgemeines Interesse. Wer \%. B., der in Sachoen lebt, stände nicht der Entwicklung, die bier das Gemeindererfassungsrecht neuerlich genommen hat, mit großer Besorgnis gegenuber und freute sich nicht, daß Sie diesem Gegenstand den morgigen Tag widmen wollen!

Drun winscben wir lhnel, indem wir Sie nochmals auls berzlichste willkommen beißen, einen recht erfolgreichen Verlauf der Tagung, Illuminiernug der Geister and Ergebnisse quasi ad inspirationem!

\section{Ansprache}

des Prüsideuten des Reichsgerichts Dr. Simons.

I eine sebrgeehrten Herren!

$\mathrm{Da} B$ die Vereinigang der deutschen Statsrechtslebrer ibre diegjihrige Tagung nacb Leipzig verlegt hat, begrubt auch das Reichsgericht mit besonderer Frende, werden doch die Beriehungen, die ansere Senate mit der Wissenschaft uber die 
staatsrechtlicheu Probleme des Reiches verbinden, von Jahr za Jahr enger; ist doch der Staatsgeriohtshof fur das Deutsche Reicb, der ein Nebenant des Reichsgerichts bildet, zeradezo auf die Verwertung der Ergebnisse Ibrer Wissenschalt angewiesen. Ob die Verbindung von Anfang an bestanden hat, kann ich nicht sagen. Als ich ror mehr als rierzig Jahren derselben juristischen Fitkultiit als Student anguhörte, die heute Ihrer Vereinigung diese wllrdigen Riume durbietct, als ich zu den Fuben des verehrten Mannes sab, dem unser Vorsitzender soeben seine Huldigung in Ihrer aller Namen dargebracht hat, da konute mav ron einer Verbindung der stiatsrechtlichen Wissenschaft mit dem Keichogericht nicht viel bemerken. Freilich kanute ich damals das Reicbsgericht fast nur in der P'erson meines ersten Vorgängers, des alten Präsidenten vou Sinsson, den ich oft in der Motette der Thonas-Kirche sah, und der in seiner Person eine reiche staitsrechtliche Erfalirung verkörperte. Jetzt ist zum Beispiel unser dhitter Senat in hervorragendem Maße mit staiatsrechtlichen Fragen beschiiftigt, und es besteht die Ans. sicht, dab das Reichsugericht dureh die Angliederung ron Verwaltungssenaten die oberste Rechtsprechung des Reichs in statutsrechtlichen Fragen erhält.

Ich will nicht den Herren Berichterstattern und der Debatte vorgreifen, aber ich balte mich doch schon jetzt fur verpflichtet, zu betonen, dab ich es fur ein Ĺngluck halten wurde, wenn ein selbstiandiges Reichsrerwaltungsgericht neben das Reichsgericht gestellt werden sollte, ein Ungluck fur das Reichsgericht und ein Unglück fur die Einheitlichkeit des dentscheu Rechtslebens. Es ist meines Erachtens ein großer Vorzug der angelstichsischen Rechtsentwicklang, daB sie offentlichrechtliche and privatrechtliche Fragen durch dieselben Gerichtsbehörden nach den einheitlicben Eutnickluugsteudenzen des British common law allezeit entschieden bat; das gibt dem angelsäcbsischen Recht bei allem Keichtum seiner Gestiltungen die innere Einheit. In Deatschland haben wir schon durch die Abzweignng des Reichsfinanzhofs vom Reichsgericht and die Eiurichtnog des Reichswirtschaftsgerichts weite Gebiete öffentlichen Kechts aus der regulierenden Tätigkeit der höchsten dentschen Instanz verloren. Die Entwicklung unseres Rechtslebens geht aber, wenn ich mich nicht irre, darauf hinaus, $\mathrm{daB}$ das öffentliche Recht sich mehr and mehr anch solcher Lebensbeziebangeu bemächtigt, die der Staat bisher der privaten Kegelung uberlieB. Ich brauche nor an die Kriegsgesetzgebung und an die Sozialisieraogstendenzen za exinnern. Wenn wir auch nicht, wie der Bolschewismus, fast jeden Unterschied zwischen priraten and offentlichen Rechtsrerbïiltuissen zngunsten der letzteren anfheben werden, so läßt sich doch nicht leugnen, daß die Ingerenz 
des Staates in das Leben seiner Burger wächst, infolgedessen wird die Entscheidung offentlichrechtlicher Fragen fur das ganze Volk immer wichtiger ond bedentsamer, während die Entscheidung vermögensrechtlicher und kriminalrechtlicher Eirzelfälle an Wichtigkeit abnimmt. Die Entleerung reichsgerichtlicher Tätigkeit von 6 ffentlichrechtlichen Gegenständen wtrde also dazn fubren, das Reichsgericht herabzudrucken and die neuen Gerichtshöfe emporsteigen za lassen. Gegen eine solche Entwicklnng maß ich meine warnende Stimme erheben.

Ich boffe, eine solche Entleerung bleibt uns erspart, and das Reichsgericht wird auch in Zukunft allen Grand haben, die Mitglieder der Vereinigung der deutschen Staatsrechtslehrer als Mitarbeiter am gleiuhen Werk begruBen za können. Ich wüssche Ibren Beratungen vollen Erfolg and wurde mich frenen, wenn Sie sich morgen abend in den Känmen des Reichsgerichto mit dessen Präsidinm and Mitgliedern, insbesondere denjenigen des dritten Zivilsenats and des Staategerichtshofs fur das Deatsche Reich, gesellig zusammenfinden wollten. 